\title{
Spatiotemporal patterns in the Lengyel-Epstein reaction-diffusion model
}

\author{
Yaying Dong ${ }^{1}$, Shunli Zhang ${ }^{1}$ and Shanbing $\mathrm{Li}^{2 *}$
}

\section{"Correspondence:}

lishanbing2006@163.com

${ }^{2}$ College of Mathematics and

Information Science, Shaanxi

Normal University, Xi'an, 710062,

P.R. China

Full list of author information is

available at the end of the article

\begin{abstract}
In this paper, we continue the mathematical study started in (Jang et al. in J. Dyn. Differ. Equ. 16:297-320, 2004; Ni and Tang in Trans. Am. Math. Soc. 357:3953-3969, 2005) on the analytic aspects of the Lengyel-Epstein reaction-diffusion system. First, we further analyze the fundamental properties of nonconstant positive solutions. On the other hand, we continue to consider the effect of the diffusion coefficient $d$. We obtain another nonexistence result for the case of large $d$ by the implicit function theory, and investigate the direction of bifurcation solutions from $\left(u^{*}, v^{*}\right)$. These results promote the Turing patterns arising from the Lengyel-Epstein reaction-diffusion system.
\end{abstract}

Keywords: Lengyel-Epstein system; nonexistence; implicit function theory; bifurcation direction; fundamental properties

\section{Introduction}

In this paper, we continue to consider the Lengyel-Epstein reaction-diffusion system [3, 4]. This system captures the crucial feature of the CIMA reaction in an open unstirred gel reactor which gave the first experimental evidence of a Turing pattern in 1990 [5]. The Lengyel and Epstein model takes the following form:

$$
\left\{\begin{array}{l}
u_{t}=\Delta u+a-u-\frac{4 u v}{1+u^{2}}, \quad x \in \Omega, t>0, \\
v_{t}=\sigma\left[c \Delta v+b\left(u-\frac{u v}{1+u^{2}}\right)\right], \quad x \in \Omega, t>0, \\
\frac{\partial u}{\partial n}=\frac{\partial v}{\partial n}=0, \quad x \in \partial \Omega, t>0, \\
u(x, 0)=u_{0}(x)>0, \quad v(x, 0)=v_{0}(x)>0, \quad x \in \Omega,
\end{array}\right.
$$

where $\Omega \subset R^{n}(n \geq 1)$ is a bounded domain with a smooth boundary $\partial \Omega ; \Delta=\sum_{i=1}^{n} \partial^{2} / \partial x_{i}^{2}$ is the Laplace operator carrying the spatial dependence of the reaction; $u(x, t)$ and $v(x, t)$, respectively, denote the chemical concentrations of iodide $\left(\mathrm{I}^{-}\right)$and chlorite $\left(\mathrm{ClO}_{2}^{-}\right)$at time $t>0$ and a point $x \in \Omega ; a$ and $b$ are the parameters, which are relative to the feed concentrations; $c$ is the ratio of the diffusion coefficients; $\sigma>1$ is a rescaling parameter, which depends on the concentration of the starch and enlarges the effective diffusion ratio to $\sigma c$. All parameters $a, b, c$, and $\sigma$ are always assumed to be positive constants. The system (1.1) is based on the chlorite-iodide-malonic acid chemical (CIMA) reaction (see $[3,5,6]$ ). The more detailed development of the CIMA reaction model and experiments can be found in [5-7] and references therein.

(c) 2016 Dong et al. This article is distributed under the terms of the Creative Commons Attribution 4.0 International License (http://creativecommons.org/licenses/by/4.0/), which permits unrestricted use, distribution, and reproduction in any medium, provided you give appropriate credit to the original author(s) and the source, provide a link to the Creative Commons license, and indicate if changes were made. 
In this paper, we are mostly concerned with the steady-state problem corresponding to (1.1), which takes the form

$$
\left\{\begin{array}{l}
\Delta u+a-u-\frac{4 u v}{1+u^{2}}=0, \quad x \in \Omega \\
d \Delta v+u-\frac{u v}{1+u^{2}}=0, \quad x \in \Omega \\
\frac{\partial u}{\partial n}=\frac{\partial v}{\partial n}=0, \quad x \in \partial \Omega
\end{array}\right.
$$

where $d=c / b$. It is easy to see that (1.2) has a unique constant positive solution

$$
\left(u^{*}, v^{*}\right)=\left(\alpha, 1+\alpha^{2}\right)
$$

where $\alpha=a / 5$.

In the past decade, the Lengyel-Epstein reaction-diffusion system (1.1) and (1.2) have been extensively studied by several authors. For example, the authors gave various important experimental and numerical studies in $[8,9]$ and the references therein. In the one-dimensional case, Yi et al. [10], regarding $b$ as the bifurcation parameter, studied the Hopf bifurcation for both the ODE and the PDE models. In [11], they further investigated the global asymptotical behavior of constant positive solution for small $a$. Taking $b$ as the bifurcation parameter, Du and Wang [12] gave the existence of multiple spatially nonhomogeneous periodic solutions though all the parameters of the system are spatially homogeneous. Furthermore, by choosing the different bifurcation parameter, Jin et al. [13] considered the same model using similar methods to [12].

On the other hand, Ni and Tang [1,2] proposed more original and systematic works on mathematical aspects. In [2], for the better description of the structures, they considered the global bifurcation of the nonconstant steady states emanating from the simple bifurcation (i.e., the case $d_{j} \neq d_{k}$ ) by choosing $d$ as bifurcation parameter. In [1], they reported some fundamental analytic properties, and investigated the nonexistence of Turing patterns and the Turing instability. Moreover, they showed that if the parameter $a$ lies in a suitable range, then the system (1.2) possesses nonconstant steady states for large $d$. In [14], the authors still viewed the effective diffusion rate $d$ as the bifurcation parameter, and maintained the basic hypothesis on the system parameters. They studied the Turing structures, especially bifurcating from the double eigenvalue (i.e., the case $d_{j}=d_{k}$ ) by using the Lyapunov-Schmidt technique and singularity theory [15], and they further discussed the stability and multiplicity of the bifurcating solutions.

In the present paper, based on the results of $\mathrm{Ni}$ et al., we continue the analytic works of $[1,2]$ with the goal of achieving a deeper understanding of the Turing patterns operating in the system (1.2). This paper is organized as follows. In Section 2, by the implicit function theory, we consider the nonexistence result for the case of large $d$. In Section 3, we continue to analyze the fundamental properties of nonconstant positive solutions. These two sections complete the work of [1]. Finally, in Section 4, we investigate the direction of bifurcation solutions from simple eigenvalue (i.e., the case $d_{j} \neq d_{k}$ ), which promotes the results in [2].

\section{The nonexistence of nonconstant steady states}

In this section, we shall verify the nonexistence of nonconstant steady states for the case of large $d$. To this end, we recall some results in [1]. First, we state a priori estimates of upper and lower bounds for positive solutions to the problem (1.2). 
Lemma 2.1 [1] Suppose that $(u, v)=(u(x), v(x))$ is a positive solution to the problem (1.2). Then

$$
\frac{a}{5+4 a^{2}}<u<a \text { and } 1+\left(\frac{a}{5+4 a^{2}}\right)^{2}<v<1+a^{2}, \quad x \in \bar{\Omega} .
$$

The following theorem gives the nonexistence of nonconstant steady states to the problem (1.2) when $d$ is not large.

Theorem 2.1 [1] There is a positive constant $d_{0}=d_{0}\left(a, \lambda_{1}\right)$ such that the problem (1.2) does not admit a nonconstant solution for $0<d<d_{0}$, where

$$
d=d_{0}\left(a, \lambda_{1}\right)=\frac{C_{2}(a) \lambda_{1}}{4 C_{1}^{2}(a)}
$$

Here $\lambda_{1}>0$ is the first positive eigenvalue of $-\Delta$ on $\Omega$ subject to the Neumann boundary condition.

We remark that it is very involved to derive a good estimate for the positive constant $d_{0}$ obtained above for a given $a$. However, Ni and Tang obtained a much simpler estimate in a different way when $a$ is not very large.

Theorem 2.2 [1] Suppose that $a^{2} \leq 75$. Then the problem (1.2) does not admit a nonconstant solution provided that

$$
\frac{1}{d}>\frac{8 a}{5}-\frac{25}{a}
$$

In particular, there is no nonconstant solution for all $d>0$ if $a^{2} \leq 125 / 8$.

Theorem 2.1 and Theorem 2.2 give the nonexistence results for the case of not large $d$. In the following, we continue to analyze the effect of the parameter $d$ on the nonexistence of nonconstant steady states to the problem (1.2). To obtain the nonexistence result for the case of large $d$, we first give the asymptotic behavior of positive solutions to (1.2) when $d$ is sufficiently large.

Lemma 2.2 Suppose that $(u, v)=(u(x), v(x))$ is any positive solution of $(1.2)$. Then $(u, v) \rightarrow$ $\left(u^{*}, v^{*}\right)$ in $\left[C^{2}(\bar{\Omega})\right]^{2}$ as $d \rightarrow \infty$.

Proof Suppose that $a$ and $\Omega$ are fixed. By Lemma 2.1 and the standard elliptic regularity theory, we may assume that for any positive solution sequence $(u, v)$ of $(1.2)$ with respect to $d$, there exists a subsequence $\left\{\left(u_{i}, v_{i}\right)\right\}_{i=1}^{\infty}$ corresponding to $d=d_{i}$ with $d_{i} \rightarrow \infty$ as $i \rightarrow \infty$, such that $\left(u_{i}, v_{i}\right) \rightarrow(\tilde{u}, \tilde{v})$ in $\left[C^{2}(\bar{\Omega})\right]^{2}$ as $i \rightarrow \infty$.

Obviously, according to Lemma 2.1, we see that $(\tilde{u}, \tilde{v})$ is a positive solution to

$$
-\Delta \tilde{u}=a-\tilde{u}-\frac{4 \tilde{u} \tilde{v}}{1+\tilde{u}^{2}} \quad \text { in } \Omega, \quad \frac{\partial \tilde{u}}{\partial n}=0 \quad \text { on } \partial \Omega,
$$

where $\tilde{v}$ is a positive constant. By Lemma 2.1 again, we easily find that

$$
\tilde{u}<a \text { and } \tilde{v}>1+\left(\frac{a}{5+4 a^{2}}\right)^{2}
$$


in $\bar{\Omega}$. Integrating the first and second equations in (1.2) over $\Omega$ by parts, respectively, we obtain

$$
\int_{\Omega} u d x+\int_{\Omega} \frac{4 u v}{1+u^{2}} d x=a|\Omega|, \quad \int_{\Omega} \frac{u v}{1+u^{2}} d x=\int_{\Omega} u d x
$$

By (2.2), we have

$$
\int_{\Omega} u d x=\frac{a}{5}|\Omega|
$$

Hence, by Lemma 2.1, together with (2.2) and (2.3), we see that $(\tilde{u}, \tilde{v})$ satisfies

$$
\tilde{v} \int_{\Omega} \frac{\tilde{u}}{1+\tilde{u}^{2}} d x=\frac{a}{5}|\Omega|, \quad \int_{\Omega} \tilde{u} d x=\frac{a}{5}|\Omega| .
$$

Apparently, $\left(u^{*}, v^{*}\right)$ is the unique positive constant solution of (2.1) and (2.4). To show the claimed result, it remains to prove $(\tilde{u}, \tilde{v})=\left(u^{*}, v^{*}\right)$.

Assume that $(\tilde{u}, \tilde{v})$ is a nonconstant solution and we shall look for a contradiction in the following. To this end, we let $\omega=\tilde{u}-u^{*}$. Then $\omega \neq \equiv 0$ in $\Omega$. By some calculations, we find that $\omega$ satisfies

$$
-\Delta \omega+f(x) \omega=g \quad \text { in } \Omega, \quad \frac{\partial \omega}{\partial n}=0 \quad \text { on } \partial \Omega,
$$

where

$$
f(x)=1+\frac{4 \tilde{v}(1-\alpha \tilde{u})}{\left(1+\alpha^{2}\right)\left(1+\tilde{u}^{2}\right)} \quad \text { and } \quad g=\frac{4 \alpha\left(v^{*}-\tilde{v}\right)}{1+\alpha^{2}} .
$$

By the second equality in (2.4), we derive

$$
\int_{\Omega} \omega d x=\int_{\Omega} \tilde{u} d x-u^{*}|\Omega|=0
$$

which suggests that $\omega$ must change sign in $\Omega$.

On the other hand, by Lemma 2.1 again, it is easy to find that $(\tilde{u}, \tilde{v})$ satisfies the following estimates:

$$
\frac{a}{5+4 a^{2}}<\tilde{u}<a, \quad 1+\left(\frac{a}{5+4 a^{2}}\right)^{2}<\tilde{v}<1+a^{2}, \quad x \in \bar{\Omega} .
$$

As a result, we find that

$$
f(x)>1+\frac{4\left[1+\left(\frac{a}{5+4 a^{2}}\right)^{2}\right](1-\alpha a)}{\left(1+\alpha^{2}\right)\left(1+a^{2}\right)} \geq 0
$$

is equivalent to

$$
4\left(\frac{a^{2}}{5}-1\right)\left(1+\left(\frac{a}{5+4 a^{2}}\right)^{2}\right) \leq\left(1+a^{2}\right)\left(1+\frac{a^{2}}{25}\right) .
$$


This inequality is true for all $a>0$. Hence, $f(x)>0$ holds for all $x \in \bar{\Omega}$. Note that $g$ is a constant. Thus, by the strong maximum principle for elliptic equations, we see that $\omega$ must be positive or negative on $\bar{\Omega}$. This contradicts $(2.6)$. Hence, the claim $(\tilde{u}, \tilde{v})=\left(u^{*}, v^{*}\right)$ follows.

Now, by Lemma 2.2, we can apply the implicit function theorem to obtain the nonexistence result for the case of large $d$.

Theorem 2.3 If $a^{2}<125 / 3$, then there exists a large constant $d^{*}=d^{*}(a, \Omega)$, such that the problem (1.2) does not admit a nonconstant solution provided that $d>d^{*}$.

Proof First, for any positive solution $(u, v)$ of the problem (1.2), we make the following decomposition on $v$ :

$$
v=v_{1}+v_{2} \quad \text { with } \int_{\Omega} v_{1} d x=0 \text { and } v_{2} \in R_{+}^{1} \text {. }
$$

For later purposes, we state the following function spaces:

$$
W_{n}^{2,2}(\Omega)=\left\{\varphi \in W^{2,2}(\Omega) \mid \frac{\partial \varphi}{\partial n}=0 \text { on } \bar{\Omega}\right\}, \quad L_{0}^{2}(\Omega)=\left\{\varphi \in L^{2}(\Omega) \mid \int_{\Omega} \varphi d x=0\right\} \text {. }
$$

Then we consider the following system:

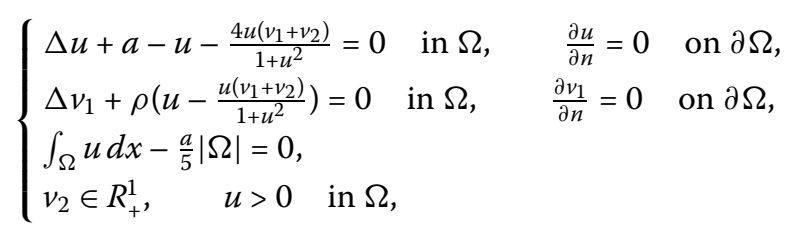

where $\rho=1 / d$. We can define the operator $F$ by

$$
F\left(\rho, u, v_{1}, v_{2}\right)=\left(f_{1}, f_{2}, f_{3}\right)\left(\rho, u, v_{1}, v_{2}\right)
$$

with

$$
\begin{aligned}
& f_{1}\left(\rho, u, v_{1}, v_{2}\right)=\Delta u+a-u-\frac{4 u\left(v_{1}+v_{2}\right)}{1+u^{2}}, \\
& f_{2}\left(\rho, u, v_{1}, v_{2}\right)=\Delta v_{1}+\rho \mathcal{P}\left(u-\frac{u\left(v_{1}+v_{2}\right)}{1+u^{2}}\right), \\
& f_{3}\left(\rho, u, v_{1}, v_{2}\right)=\int_{\Omega} u d x-\frac{a}{5}|\Omega|,
\end{aligned}
$$

where $\mathcal{P}$ is a projection operator from $L^{2}(\Omega)$ to $L_{0}^{2}(\Omega)$, which satisfies

$$
\mathcal{P}(\varphi)=\varphi-\frac{1}{|\Omega|} \int_{\Omega} \varphi d x, \quad \forall \varphi \in L^{2}(\Omega)
$$

Then, for any fixed $\rho>0$,

$$
F: W_{n}^{2,2}(\Omega) \times\left(W_{n}^{2,2}(\Omega) \cap L_{0}^{2}(\Omega)\right) \times R_{+}^{1} \rightarrow L^{2}(\Omega) \times L_{0}^{2}(\Omega) \times R^{1}
$$


is a well-defined mapping. For any given $\rho>0,\left(u, v_{1}, v_{2}\right)$ is a solution of (2.7) if and only if $F\left(\rho, u, v_{1}, v_{2}\right)=(0,0,0)$. Apparently, for all $\rho \geq 0,\left(u, v_{1}, v_{2}\right)=\left(u^{*}, 0, v^{*}\right)$ is a solution of $(2.7)$. In addition, by the standard elliptic regularity theory, we easily check that the positive solutions $(u, v)$ of $(1.2)$ are equivalent to the roots of $F\left(\rho, u, v_{1}, v_{2}\right)=(0,0,0)$.

It is obvious that $F$ is a continuously differentiable mapping. By some calculations, we see that the partial derivative of $F$ at the point $\left(0, u^{*}, 0, v^{*}\right)$ with respect to $\left(u, v_{1}, v_{2}\right)$ is provided by

$$
\mathcal{L}:=D_{\left(u, v_{1}, v_{2}\right)} F\left(0, u^{*}, 0, v^{*}\right)
$$

where

$$
\mathcal{L}: W_{n}^{2,2}(\Omega) \times\left(W_{n}^{2,2}(\Omega) \cap L_{0}^{2}(\Omega)\right) \times R_{+}^{1} \rightarrow L^{2}(\Omega) \times L_{0}^{2}(\Omega) \times R^{1}
$$

with

$$
\mathcal{L}(\omega, z, \tau)=\left[\begin{array}{c}
\Delta \omega+\frac{3 \alpha^{2}-5}{1+\alpha^{2}} \omega-\frac{4 \alpha}{1+\alpha^{2}}(z+\tau) \\
\Delta z \\
\int_{\Omega} \omega d x
\end{array}\right] .
$$

In the following, we verify that $\mathcal{L}$ is an isomorphism. First of all, we claim that $\mathcal{L}$ is injective. Assume that $\mathcal{L}(\omega, z, \tau)=(0,0,0)$. Since the operator $-\Delta$, subject to the homogeneous Neumann boundary condition over $\partial \Omega$, is invertible from $W_{n}^{2,2}(\Omega) \cap L_{0}^{2}(\Omega)$ to $L_{0}^{2}(\Omega)$, we have $z=0$. Thus, we obtain

$$
-\Delta \omega-\frac{3 \alpha^{2}-5}{1+\alpha^{2}} \omega=-\frac{4 \alpha}{1+\alpha^{2}} \tau \quad \text { in } \Omega, \quad \frac{\partial \omega}{\partial n}=0 \quad \text { on } \partial \Omega .
$$

When $a^{2}<125 / 3$, we can observe that $5-3 \alpha^{2}>0$. Hence, for any given constant $\tau$, for equation (2.9) there exists a unique solution,

$$
\omega=\frac{4 \alpha}{3 \alpha^{2}-5} \tau
$$

On the other hand, in Lemma 2.2, we have proved that $\omega$ satisfies

$$
\int_{\Omega} \omega d x=0
$$

Combining this with (2.10), we can derive $\omega=\tau=0$. So $\mathcal{L}$ is injective.

On the other hand, we need to prove that $\mathcal{L}$ is surjective. To see that $\mathcal{L}$ is surjective, we just need an elementary fact from [16]: for any given function $g \in L_{0}^{2}(\Omega)$ and constant $\tau_{0}$, the elliptic equation

$$
-\Delta \omega=g \quad \text { in } \Omega, \quad \frac{\partial \omega}{\partial n}=0 \quad \text { on } \partial \Omega \quad \text { with } \int_{\Omega} \omega d x=\tau_{0},
$$

has a unique solution $\omega \in W_{n}^{2,2}(\Omega)$. As before, together with the previous fact, it is not hard to show that $\mathcal{L}$ is surjective. 
By virtue of the implicit function theorem, we see that there exist two positive constants $\rho_{0}$ and $r_{0}$, which are sufficiently small, such that for any $\rho \in\left(0, \rho_{0}\right],\left(u^{*}, 0, v^{*}\right)$ is the unique solution of $F\left(\rho, u, v_{1}, v_{2}\right)=0$ in $B_{r_{0}}\left(u^{*}, 0, v^{*}\right)$, where $B_{r_{0}}\left(u^{*}, 0, v^{*}\right)$ denotes the open ball in $W_{n}^{2,2}(\Omega) \times\left(W_{n}^{2,2}(\Omega) \cap L_{0}^{2}(\Omega)\right) \times R_{+}^{1}$ centered at $\left(u^{*}, 0, v^{*}\right)$ with radius $r_{0}$.

For any positive constant sequence $\left\{\rho_{i}\right\}_{i=1}^{\infty}$ satisfying $\rho_{i} \rightarrow 0$ as $i \rightarrow \infty$, we let $\left(u_{i}, v_{i}\right)$ be a corresponding solution sequence of (1.2) for $d_{i}=1 / \rho_{i}$ with the decomposition

$$
v_{i}=v_{i, 1}+v_{i, 2}, \quad \text { where } \int_{\Omega} v_{i, 1} d x=0 \text { and } v_{i, 2} \in R_{+}^{1} \text { for each } i \geq 1 \text {. }
$$

Obviously, $\left(\rho_{i}, u_{i}, v_{i, 1}, v_{i, 2}\right)$ is a root of

$$
F\left(\rho_{i}, u_{i}, v_{i, 1}, v_{i, 2}\right)=(0,0,0)
$$

By virtue of Lemma 2.2 and our decomposition, we have

$$
\left(u_{i}, v_{i, 1}, v_{i, 2}\right) \rightarrow\left(u^{*}, 0, v^{*}\right) \quad \text { as } i \rightarrow \infty
$$

It follows from (2.11) that for all large $i$,

$$
\left(\rho_{i}, u_{i}, v_{i, 1}, v_{i, 2}\right) \in\left(0, \rho_{0}\right) \times B_{r_{0}}\left(u^{*}, 0, v^{*}\right) .
$$

In view of the implicit function theorem, we obtain

$$
\left(u_{i}, v_{i, 1}, v_{i, 2}\right)=\left(u^{*}, 0, v^{*}\right)
$$

that is, if $d$ is sufficiently large, then $\left(u^{*}, v^{*}\right)$ is the unique positive solution of (1.2).

\section{Properties of nonconstant positive solutions}

In the section, based on the results of $\mathrm{Ni}$ and Tang [1], we continue to investigate the basic properties of nonconstant positive solutions to the Lengyel-Epstein reaction-diffusion system.

For any given positive solution $(u, v)=(u(x), v(x))$ to the problem (1.2), we denote their averages over $\Omega$ by

$$
\bar{u}=\frac{1}{|\Omega|} \int_{\Omega} u d x \text { and } \bar{v}=\frac{1}{|\Omega|} \int_{\Omega} v d x
$$

where $|\Omega|$ is the volume of $\Omega$. Let $\phi=u-\bar{u}, \psi=v-\bar{v}$. Then

$$
\int_{\Omega} \phi d x=\int_{\Omega} \psi d x=0
$$

Lemma 2.1 guarantees that there exist two positive constants $M_{1}$ and $M_{2}$, which depend, respectively, only on $a$, such that

$$
f(u, v)=\left|a-u-\frac{4 u v}{1+u^{2}}\right| \leq M_{1}, \quad g(u, v)=\left|u-\frac{u v}{1+u^{2}}\right| \leq M_{2} .
$$

This enables us to derive the following two lemmas. 
Lemma 3.1 There exists a constant $M_{2}$ depending only on a, such that

$$
\int_{\Omega}|\nabla \psi|^{2} d x \leq \frac{M_{2}^{2}|\Omega|}{d^{2} \lambda_{1}} \quad \text { and } \quad \int_{\Omega}|\psi|^{2} d x \leq \frac{M_{2}^{2}|\Omega|}{d^{2} \lambda_{1}^{2}} .
$$

Proof By means of Lemma 2.1 and the Hölder inequality, we get

$$
d \int_{\Omega}|\nabla \psi|^{2} d x=\int_{\Omega}\left(u-\frac{u v}{1+u^{2}}\right) \psi d x \leq M_{2} \int_{\Omega}|\psi| d x \leq M_{2}|\Omega|^{\frac{1}{2}}\left(\int_{\Omega}|\psi|^{2} d x\right)^{\frac{1}{2}} .
$$

By the Poincaré inequality

$$
\int_{\Omega} \psi^{2} d x \leq \frac{1}{\lambda_{1}} \int_{\Omega}|\nabla \psi|^{2} d x
$$

where $\lambda_{1}(>0)$ is the first positive eigenvalue of $-\Delta$ subject to the Neumann boundary condition, we obtain

$$
d \int_{\Omega}|\nabla \psi|^{2} d x \leq M_{2}|\Omega|^{\frac{1}{2}} \frac{1}{\sqrt{\lambda_{1}}}\left(\int_{\Omega}|\nabla \psi|^{2} d x\right)^{\frac{1}{2}}=M_{2} \sqrt{|\Omega| / \lambda_{1}}\left(\int_{\Omega}|\nabla \psi|^{2} d x\right)^{\frac{1}{2}} .
$$

This implies

$$
\int_{\Omega}|\nabla \psi|^{2} d x \leq \frac{M_{2}^{2}|\Omega|}{d^{2} \lambda_{1}}
$$

Applying the Poincaré inequality again, we derive

$$
\int_{\Omega}|\psi|^{2} d x \leq \frac{1}{\lambda_{1}} \int_{\Omega}|\nabla \psi|^{2} d x \leq \frac{M_{2}^{2}|\Omega|}{d^{2} \lambda_{1}^{2}} .
$$

We complete the proof of the lemma.

Lemma 3.2 There exists a constant $M_{1}$ depending only on a, such that

$$
\int_{\Omega}|\nabla \phi|^{2} d x \leq \frac{M_{1}^{2}|\Omega|}{\lambda_{1}} \text { and } \int_{\Omega}|\phi|^{2} d x \leq \frac{M_{1}^{2}|\Omega|}{\lambda_{1}^{2}} .
$$

Proof By means of Lemma 2.1 and the Hölder inequality, we obtain

$$
\int_{\Omega}|\nabla \phi|^{2} d x=\int_{\Omega}\left(a-u-\frac{4 u v}{1+u^{2}}\right) \phi d x \leq M_{1} \int_{\Omega}|\phi| d x \leq M_{1}|\Omega|^{\frac{1}{2}}\left(\int_{\Omega}|\phi|^{2} d x\right)^{\frac{1}{2}} .
$$

By the Poincaré inequality

$$
\int_{\Omega} \phi^{2} d x \leq \frac{1}{\lambda_{1}} \int_{\Omega}|\nabla \phi|^{2} d x
$$

where $\lambda_{1}(>0)$ is the first positive eigenvalue of $-\Delta$ subject to the Neumann boundary condition, we derive

$$
\int_{\Omega}|\nabla \phi|^{2} d x \leq M_{1}|\Omega|^{\frac{1}{2}}\left(\int_{\Omega}|\phi|^{2} d x\right)^{\frac{1}{2}} \leq M_{1} \sqrt{|\Omega| / \lambda_{1}}\left(\int_{\Omega}|\nabla \phi|^{2} d x\right)^{\frac{1}{2}} .
$$


This suggests

$$
\int_{\Omega}|\nabla \phi|^{2} d x \leq \frac{M_{1}^{2}|\Omega|}{\lambda_{1}}
$$

Applying the Poincaré inequality again, we have

$$
\int_{\Omega}|\phi|^{2} d x \leq \frac{1}{\lambda_{1}} \int_{\Omega}|\nabla \phi|^{2} d x \leq \frac{M_{1}^{2}|\Omega|}{\lambda_{1}^{2}}
$$

The proof is complete.

By (3.1) and (3.2), we have the following theorem.

Theorem 3.1 Suppose that $(u, v)$ is a nonconstant solution of the problem (1.2). Then the following estimates hold:

$$
\|\phi\|_{W^{1,2}(\Omega)} \leq M_{1}|\Omega|^{\frac{1}{2}}\left(\frac{1}{\lambda_{1}}+\frac{1}{\sqrt{\lambda_{1}}}\right) \quad \text { and } \quad\|\psi\|_{W^{1,2}(\Omega)} \leq \frac{M_{2}|\Omega|^{\frac{1}{2}}}{d}\left(\frac{1}{\lambda_{1}}+\frac{1}{\sqrt{\lambda_{1}}}\right) .
$$

Next, we promote the relationship of the gradients of $u$ and $v$ based on the work of Ni and Tang. It is to be found that our proof does not depend on the previous estimates. For this purpose, we need to introduce some results.

Lemma 3.3 [1] Suppose that $(u, v)$ is a nonconstant solution of the problem (1.2). Then

$$
\frac{12 \lambda_{1}^{2}}{3 \lambda_{1}^{2}+15 \lambda_{1}+25}<\frac{\int_{\Omega}|\nabla u|^{2} d x}{d^{2} \int_{\Omega}|\nabla v|^{2} d x}<16
$$

Lemma 3.4 [1] Suppose that $(u, v)$ is a nonconstant solution of the problem (1.2). Then

$$
\frac{12 \lambda_{1}^{3}}{\left(\lambda_{1}+1\right)\left(3 \lambda_{1}^{2}+15 \lambda_{1}+25\right)}<\frac{\int_{\Omega}\left(|\nabla \phi|^{2}+\phi^{2}\right) d x}{d^{2} \int_{\Omega}\left(|\nabla \psi|^{2}+\psi^{2}\right) d x}<16
$$

Now, by Lemma 3.3 and Lemma 3.4, our purpose is to obtain a new result on the relationship of the gradients of $u$ and $v$.

Theorem 3.2 Suppose that $(u, v)$ be a nonconstant solution of the problem (1.2). Then

$$
\frac{12 \lambda_{1}^{3}}{\left(\lambda_{1}+1\right)\left(3 \lambda_{1}^{2}+15 \lambda_{1}+25\right)}<\frac{\int_{\Omega}\left(|\nabla \phi|^{2}+c \phi^{2}\right) d x}{d^{2} \int_{\Omega}\left(|\nabla \psi|^{2}+\psi^{2}\right) d x}<16
$$

where $0 \leq c \leq 10$.

Proof Let

$$
w(x)=4 d v(x)-u(x)
$$

Then $w(x)$ satisfies

$$
\Delta w-a+5 u=0 .
$$


Multiplying $\phi$ and integrating by parts in the above equation, we obtain

$$
5 \int_{\Omega} \phi^{2} d x=\int_{\Omega} \nabla \phi \nabla w d x=4 d \int_{\Omega} \nabla \phi \nabla \psi d x-\int_{\Omega}|\nabla \phi|^{2} d x,
$$

which suggests that

$$
\int_{\Omega} \nabla \phi \nabla \psi d x=\frac{1}{4 d}\left(\int_{\Omega}|\nabla \phi|^{2} d x+5 \int_{\Omega} \phi^{2} d x\right) .
$$

By (3.5), we get

$$
\begin{aligned}
\int_{\Omega}|\nabla w|^{2} d x & =16 d^{2} \int_{\Omega}|\nabla v|^{2} d x-8 d \int_{\Omega}|\nabla v| \cdot|\nabla u| d x+\int_{\Omega}|\nabla u|^{2} d x \\
& =16 d^{2} \int_{\Omega}|\nabla \psi|^{2} d x-8 d \int_{\Omega}|\nabla \psi| \cdot|\nabla \phi| d x+\int_{\Omega}|\nabla \phi|^{2} d x \\
& =16 d^{2} \int_{\Omega}|\nabla \psi|^{2} d x-\int_{\Omega}|\nabla \phi|^{2} d x-10 \int_{\Omega} \phi^{2} d x
\end{aligned}
$$

Hence

$$
\int_{\Omega}|\nabla \phi|^{2} d x+10 \int_{\Omega} \phi^{2} d x \leq 16 d^{2} \int_{\Omega}|\nabla \psi|^{2} d x .
$$

By the Poincaré inequality, we have

$$
\int_{\Omega}\left(|\nabla \psi|^{2}+\psi^{2}\right) d x \leq \frac{\lambda_{1}+1}{\lambda_{1}} \int_{\Omega}|\nabla \psi|^{2} d x .
$$

By the first inequality of (3.3), we have

$$
\frac{\int_{\Omega}\left(|\nabla \phi|^{2}+c \phi^{2}\right) d x}{d^{2} \int_{\Omega}\left(|\nabla \psi|^{2}+\psi^{2}\right) d x} \geq \frac{\lambda_{1} \int_{\Omega}|\nabla \phi|^{2} d x}{d^{2}\left(\lambda_{1}+1\right) \int_{\Omega}|\nabla \psi|^{2} d x}>\frac{12 \lambda_{1}^{3}}{\left(\lambda_{1}+1\right)\left(3 \lambda_{1}^{2}+15 \lambda_{1}+25\right)} .
$$

On the other hand, by (3.6), we have

$$
\frac{\int_{\Omega}\left(|\nabla \phi|^{2}+c \phi^{2}\right) d x}{d^{2} \int_{\Omega}\left(|\nabla \psi|^{2}+\psi^{2}\right) d x}<\frac{\int_{\Omega}\left(|\nabla \phi|^{2}+10 \phi^{2}\right) d x}{d^{2} \int_{\Omega}|\nabla \psi|^{2} d x}<\frac{16 d^{2} \int_{\Omega}|\nabla \psi|^{2} d x}{d^{2} \int_{\Omega}|\nabla \psi|^{2} d x}=16 .
$$

Therefore, we finish the proof of this theorem.

\section{Direction of the bifurcation solutions}

In [2], Ni et al. derived the local and global bifurcation from $\left(u^{*}, v^{*}\right)$ in one-dimensional spatial domain $\Omega=(0, l)$. That is, they obtained the local and global bifurcation from $\left(u^{*}, v^{*}\right)$ to the following problem:

$$
\left\{\begin{array}{l}
u_{x x}+a-u-\frac{4 u v}{1+u^{2}}=0, \quad x \in(0, l) \\
d v_{x x}+u-\frac{u v}{1+u^{2}}=0, \quad x \in(0, l) \\
u_{x}(0, t)=v_{x}(0, t)=u_{x}(l, t)=v_{x}(l, t)=0
\end{array}\right.
$$


In this section, we follow the idea of Shi [17] to determine the bifurcation direction of the steady-state bifurcation from simple eigenvalue. To this end, we translate (1.2) into the following system by the transition $\hat{u}=u-u^{*}$ and $\hat{v}=v-v^{*}$. For the sake of convenience, we still denote $(\hat{u}, \hat{v})$ by $(u, v)$, then we have

$$
\left\{\begin{array}{l}
u_{x x}+4 \alpha-u-\frac{4(u+\alpha)\left(v+1+\alpha^{2}\right)}{1+(u+\alpha)^{2}}=0, \quad x \in(0, \pi), \\
d v_{x x}+u+\alpha-\frac{(u+\alpha)\left(v+1+\alpha^{2}\right)}{1+(u+\alpha)^{2}}=0, \quad x \in(0, \pi), \\
u_{x}(0, t)=v_{x}(0, t)=u_{x}(\pi, t)=v_{x}(\pi, t)=0
\end{array}\right.
$$

where we denote $l=\pi$. Let

$$
\begin{aligned}
& \tilde{f}(u, v)=4 \alpha-u-\frac{4(u+\alpha)\left(v+1+\alpha^{2}\right)}{1+(u+\alpha)^{2}}, \\
& \tilde{g}(u, v)=u+\alpha-\frac{(u+\alpha)\left(v+1+\alpha^{2}\right)}{1+(u+\alpha)^{2}} .
\end{aligned}
$$

A direct calculation yields

$$
\left\{\begin{array}{l}
\tilde{f}_{u}(0,0)=\frac{3 \alpha^{2}-5}{1+\alpha^{2}}, \quad \tilde{f}_{v}(0,0)=\frac{-4 \alpha}{1+\alpha^{2}} \\
\tilde{g}_{u}(0,0)=\frac{2 \alpha^{2}}{1+\alpha^{2}}, \quad \tilde{g}_{v}(0,0)=\frac{-\alpha}{1+\alpha^{2}}, \\
\tilde{f}_{u u}(0,0)=4 \tilde{g}_{u u}(0,0)=\frac{8 \alpha\left(3-\alpha^{2}\right)}{\left(1+\alpha^{2}\right)^{2}} \\
\tilde{f}_{u v}(0,0)=4 \tilde{g}_{u v}(0,0)=-\frac{4\left(1-\alpha^{2}\right)}{\left(1+\alpha^{2}\right)^{2}} \\
\tilde{f}_{v v}(0,0)=\tilde{g}_{v v}(0,0)=0 \\
\tilde{f}_{u u u}(0,0)=4 \tilde{g}_{u u u}(0,0)=\frac{24\left(\alpha^{4}-6 \alpha^{2}+1\right)}{\left(1+\alpha^{2}\right)^{3}} \\
\tilde{f}_{u u v}(0,0)=4 \tilde{g}_{u u v}(0,0)=\frac{8 \alpha\left(3-\alpha^{2}\right)}{\left(1+\alpha^{2}\right)^{3}}, \\
\tilde{f}_{u v v}(0,0)=\tilde{g}_{u v v}(0,0)=\tilde{f}_{v v v}(0,0)=\tilde{g}_{v v v}(0,0)=0 .
\end{array}\right.
$$

Denote $U=(u, v)$. We define the map $\widetilde{F}: R^{+} \times X \rightarrow Y$ by

$$
\widetilde{F}(d, U)=\left[\begin{array}{c}
u^{\prime \prime}+\tilde{f}(u, v) \\
d v^{\prime \prime}+\tilde{g}(u, v)
\end{array}\right]
$$

where $X$ is a Banach space with usual $C^{2}$ norm and $Y=L^{2}(0, \pi) \times L^{2}(0, \pi)$. By virtue of Theorem 3 in [2], we see that $\operatorname{dim} \operatorname{ker} \widetilde{F}_{U}\left(d_{j},(0,0)\right)=\operatorname{codim} R\left(\widetilde{F}_{U}\left(d_{j},(0,0)\right)\right)=1$ and $\operatorname{ker} \widetilde{F}_{U}\left(d_{j},(0,0)\right)=\operatorname{span}\left\{\Phi_{j}\right\}$, where

$$
\Phi_{j}=\left[\begin{array}{c}
1 \\
e_{j}
\end{array}\right] \phi_{j}
$$

with

$$
e_{j}=\frac{3 \alpha^{2}-5-\left(1+\alpha^{2}\right) j^{2}}{4 \alpha}>0 \text { and } \phi_{j}=\cos j x
$$

Hence, we can decompose $X$ and $Y$ as

$$
X=\operatorname{ker} \widetilde{F}_{U}\left(d_{j},(0,0)\right) \oplus Z \quad \text { and } \quad Y=R\left(\widetilde{F}_{U}\left(d_{j},(0,0)\right)\right) \oplus Z^{\prime}
$$


where $Z$ is the complement of $\operatorname{ker} \widetilde{F}_{U}(d,(0,0))$ in $X$ and $Z^{\prime}$ is the complement of $R\left(\widetilde{F}_{U}(d,(0\right.$, $0)))$ in $Y$. Due to $\operatorname{codim} R\left(\widetilde{F}_{U}\left(d_{j},(0,0)\right)\right)=1$, there exists $l \in Y^{*}$ such that

$$
R\left(\widetilde{F}_{U}\left(d_{j},(0,0)\right)\right)=\{(\xi, \zeta) \in Y:\langle l,(\xi, \zeta)\rangle=0\} .
$$

Moreover, $\Phi_{j}^{*}$ satisfies $\widetilde{F}_{U}^{*}\left(d_{j},(0,0)\right) \Phi_{j}^{*}=0$ by Theorem 3 in [2] again, where

$$
\Phi_{j}^{*}=\left[\begin{array}{c}
1 \\
e_{j}^{*}
\end{array}\right] \phi_{j} \quad \text { and } \quad e_{j}^{*}=-\frac{3 \alpha^{2}-5-\left(1+\alpha^{2}\right) j^{2}}{2 \alpha^{2}}<0 .
$$

Thus, we can define

$$
\langle l,(\xi, \zeta)\rangle=\left\langle\Phi_{j}^{*},(\xi, \zeta)\right\rangle=\int_{\Omega} \xi \phi_{j} d x+\int_{\Omega} e_{j}^{*} \zeta \phi_{j} d x
$$

Since $\widetilde{F}_{d U}\left(d_{j},(0,0)\right) \Phi_{j} \notin R\left(\widetilde{F}_{U}\left(d_{j},(0,0)\right)\right)$, we find that

$$
\left\langle\widetilde{F}_{d u}\left(d_{j},(0,0)\right) \Phi_{j}, \Phi_{j}^{*}\right\rangle \neq 0 \text {. }
$$

According to (4.5) in [17], we see that

$$
d^{\prime}(0)=-\frac{\left\langle\widetilde{F}_{U U}\left(d_{j},(0,0)\right) \Phi_{j}^{2}, \Phi_{j}^{*}\right\rangle}{2\left\langle\widetilde{F}_{d U}\left(d_{j},(0,0)\right) \Phi_{j}, \Phi_{j}^{*}\right\rangle} .
$$

By some calculations, we obtain

$$
\begin{aligned}
\left\langle\widetilde{F}_{U u}\left(d_{j},(0,0)\right) \Phi_{j}^{2}, \Phi_{j}^{*}\right\rangle & =\left(g_{j}+h_{j} e_{j}^{*}\right) \int_{\Omega} \phi_{j}^{3} d x=0, \\
\left\langle\widetilde{F}_{d U}\left(d_{j},(0,0)\right) \Phi_{j}, \Phi_{j}^{*}\right\rangle & =\int_{0}^{\pi} e_{j}^{*} \cos j x \Delta\left(e_{j} \cos j x\right) d x=-\frac{\pi j^{2}}{2} e_{j} e_{j}^{*},
\end{aligned}
$$

where

$$
\left\{\begin{array}{l}
g_{j}=\tilde{f}_{u u}(0,0)+2 \tilde{f}_{u v}(0,0) e_{j}+\tilde{f}_{v v}(0,0) e_{j}^{2} \\
h_{j}=\tilde{g}_{u u}(0,0)+2 \tilde{g}_{u v}(0,0) e_{j}+\tilde{g}_{v v}(0,0) e_{j}^{2}
\end{array}\right.
$$

By (4.2), we obtain

$$
g_{j}=4 h_{j}=\tilde{f}_{u u}(0,0)+2 \tilde{f}_{u v}(0,0) e_{j} .
$$

Hence, $d^{\prime}(0)=0$.

Also from [17], we see that the bifurcation is supercritical (resp. subcritical) provided that

$$
d^{\prime \prime}(0)=-\frac{\left\langle\widetilde{F}_{u u u}\left(d_{j},(0,0)\right) \Phi_{j}^{3}, \Phi_{j}^{*}\right\rangle+3\left\langle\widetilde{F}_{U u}\left(d_{j},(0,0)\right) \Phi_{j} \theta, \Phi_{j}^{*}\right\rangle}{3\left\langle\widetilde{F}_{U u}\left(d_{j},(0,0)\right) \Phi_{j}, \Phi_{j}^{*}\right\rangle}>0(<0),
$$

where $\theta$ is the solution of the following problem:

$$
\widetilde{F}_{U U}\left(d_{j},(0,0)\right) \Phi_{j}^{2}+\widetilde{F}_{U}\left(d_{j},(0,0)\right) \theta=0 .
$$


Let $\theta=\left(\theta_{1}, \theta_{2}\right)$. Then $\theta$ satisfies

$$
\left\{\begin{array}{l}
\Delta \theta_{1}+\tilde{f}_{u}(0,0) \theta_{1}+\tilde{f}_{v}(0,0) \theta_{2}=-g_{j} \cos ^{2} j x \\
d \Delta \theta_{2}+\tilde{g}_{u}(0,0) \theta_{1}+\tilde{g}_{v}(0,0) \theta_{2}=-h_{j} \cos ^{2} j x \\
\theta_{i}(0, t)=\theta_{i}(\pi, t)=0, \quad i=1,2
\end{array}\right.
$$

By direct calculation, we obtain

$$
\left\langle\widetilde{F}_{\text {UuU }}\left(d_{j},(0,0)\right) \Phi_{j}^{3}, \Phi_{j}^{*}\right\rangle=\left(m_{j}+n_{j} e_{j}^{*}\right) \int_{0}^{\pi} \cos ^{4} j x d x=\frac{3 \pi}{8}\left(m_{j}+n_{j} e_{j}^{*}\right),
$$

where

$$
\left\{\begin{array}{l}
m_{j}=\tilde{f}_{u u u}(0,0)+3 e_{j} \tilde{f}_{u u v}(0,0)+3 e_{j}^{2} \tilde{f}_{u v v}(0,0)+\tilde{f}_{v v v}(0,0), \\
n_{j}=\tilde{g}_{u u u}(0,0)+3 e_{j} \tilde{g}_{u u v}(0,0)+3 e_{j}^{2} \tilde{g}_{u v v}(0,0)+\tilde{g}_{v v v}(0,0) .
\end{array}\right.
$$

By (4.2) again, we have

$$
m_{j}=4 n_{j}=\tilde{f}_{\text {uuu }}(0,0)+3 e_{j} \tilde{f}_{\text {uuv }}(0,0) .
$$

Hence

$$
\left\langle\widetilde{F}_{\text {uиu }}\left(d_{j},(0,0)\right) \Phi_{j}^{3}, \Phi_{j}^{*}\right\rangle=\frac{3 \pi}{8}\left(1+\frac{1}{4} e_{j}^{*}\right)\left(\tilde{f}_{\text {uuu }}(0,0)+3 e_{j} \tilde{f}_{\text {uuv }}(0,0)\right) .
$$

On the other hand, we have

$$
\left\langle\widetilde{F}_{U U}\left(d_{j},(0,0)\right) \Phi_{j} \theta, \Phi_{j}^{*}\right\rangle=C_{1} \int_{0}^{\pi} \theta_{1} \cos ^{2} j x d x+C_{2} \int_{0}^{\pi} \theta_{2} \cos ^{2} j x d x,
$$

where

$$
\begin{aligned}
C_{1} & =\tilde{f}_{u u}(0,0)+e_{j} \tilde{f}_{u v}(0,0)+e_{j}^{*} \tilde{g}_{u u}(0,0)+e_{j} e_{j}^{*} \tilde{g}_{u v}(0,0) \\
& =\tilde{f}_{u u}(0,0)\left(1+\frac{1}{4} e_{j}^{*}\right)+\tilde{f}_{u v}(0,0) e_{j}\left(1+\frac{1}{4} e_{j}^{*}\right), \\
C_{2} & =\tilde{f}_{u v}(0,0)+e_{j} \tilde{f}_{v v}(0,0)+e_{j}^{*} \tilde{g}_{u v}(0,0)+e_{j} e_{j}^{*} \tilde{g}_{v v}(0,0) \\
& =\tilde{f}_{u v}(0,0)\left(1+\frac{1}{4} e_{j}^{*}\right) .
\end{aligned}
$$

In the following, we shall compute

$$
\int_{0}^{\pi} \theta_{1} \cos ^{2} j x d x \text { and } \int_{0}^{\pi} \theta_{2} \cos ^{2} j x d x
$$

Multiplying (4.3) by $\cos ^{2} j x$ and integrating by parts, we derive

$$
\left\{\begin{array}{c}
\int_{0}^{\pi} \cos ^{2} j x \Delta \theta_{1} d x+\tilde{f}_{u}(0,0) \int_{0}^{\pi} \cos ^{2} j x \theta_{1} d x \\
\quad+\tilde{f}_{v}(0,0) \int_{0}^{\pi} \cos ^{2} j x \theta_{2} d x=-g_{j} \int_{0}^{\pi} \cos ^{4} j x d x \\
d \int_{0}^{\pi} \cos ^{2} j x \Delta \theta_{2} d x+\tilde{g}_{u}(0,0) \int_{0}^{\pi} \cos ^{2} j x \theta_{1} d x \\
\quad+\tilde{g}_{v}(0,0) \int_{0}^{\pi} \cos ^{2} j x \theta_{2} d x=-h_{j} \int_{0}^{\pi} \cos ^{4} j x d x
\end{array}\right.
$$


where

$$
\int_{0}^{\pi} \cos ^{2} j x \Delta \theta_{i} d x=2 j^{2} \int_{0}^{\pi} \theta_{i}\left(1-2 \cos ^{2} j x\right) d x, \quad i=1,2 .
$$

Furthermore, integrating (4.3) by parts, we have

$$
\begin{aligned}
& \gamma_{1} \triangleq \int_{0}^{\pi} \theta_{1} d x=\frac{\pi\left(h_{j} \tilde{f}_{v}(0,0)-g_{j} \tilde{g}_{v}(0,0)\right)}{2\left(\tilde{f}_{u}(0,0) \tilde{g}_{v}(0,0)-\tilde{f}_{v}(0,0) \tilde{g}_{u}(0,0)\right)}=0, \\
& \gamma_{2} \triangleq \int_{0}^{\pi} \theta_{2} d x=\frac{\pi\left(g_{j} \tilde{g}_{u}(0,0)-h_{j} \tilde{f}_{u}(0,0)\right)}{2\left(\tilde{f}_{u}(0,0) \tilde{g}_{v}(0,0)-\tilde{f}_{v}(0,0) \tilde{g}_{u}(0,0)\right)}=-\frac{\pi g_{j}}{2 \tilde{f}_{v}(0,0)} .
\end{aligned}
$$

By (4.4), it is not hard to check that

$$
\left\{\begin{array}{l}
\left(\tilde{f}_{u}(0,0)-4 j^{2}\right) \int_{0}^{\pi} \cos ^{2} j x \theta_{1} d x+\tilde{f}_{v}(0,0) \int_{0}^{\pi} \cos ^{2} j x \theta_{2} d x=-\frac{3 g_{j} \pi}{8}-2 \gamma_{1} j^{2} \\
\left(\tilde{g}_{v}(0,0)-4 d j^{2}\right) \int_{0}^{\pi} \cos ^{2} j x \theta_{2} d x+\tilde{g}_{u}(0,0) \int_{0}^{\pi} \cos ^{2} j x \theta_{1} d x=-\frac{3 h_{j} \pi}{8}-2 d \gamma_{2} j^{2} .
\end{array}\right.
$$

Therefore, it follows that

$$
\begin{aligned}
& L_{1} \triangleq \int_{0}^{\pi} \theta_{1} \cos ^{2} j x d x=\frac{-\frac{3 d \pi j^{2}}{2} g_{j}-2 d j^{2} \gamma_{2} \tilde{f}_{v}(0,0)}{\tilde{f}_{v}(0,0)+4 d j^{2} \tilde{f}_{u}(0,0)+4 j^{2} \tilde{f}_{v}(0,0)-16 d j^{4}}, \\
& L_{2} \triangleq \int_{0}^{\pi} \theta_{2} \cos ^{2} j x d x=\frac{-\frac{3 \pi}{8} g_{j}-\frac{3 j^{2} \pi}{2} h_{j}+2 d j^{2} \gamma_{2} \tilde{f}_{u}(0,0)-8 d \gamma_{2} j^{4}}{\tilde{f}_{v}(0,0)+4 d j^{2} \tilde{f}_{u}(0,0)+4 j^{2} \tilde{f}_{v}(0,0)-16 d j^{4}} .
\end{aligned}
$$

Hence

$$
d^{\prime \prime}(0)=-\frac{\pi\left(1+e_{j}^{*}\right)\left(\tilde{f}_{\text {uиu }}(0,0)+3 e_{j} \tilde{f}_{\text {uuv }}(0,0)\right)+8\left(C_{1} L_{1}+C_{2} L_{2}\right)}{4 j^{2} \pi e_{j} e_{j}^{*}} .
$$

From the above analysis, we obtain the following results.

Theorem 4.1 Under the same hypothesis as Theorem 3 in [2], there exists a smooth bifurcation branch from $\left(d_{j},(0,0)\right)$. Furthermore, the bifurcation is supercritical (resp. subcritical) provided that $d^{\prime \prime}(0)>0(<0)$, where $d^{\prime \prime}(0)$ is given by $(4.5)$.

\section{Conclusion}

In this paper, we have studied the Lengyel-Epstein reaction-diffusion system which is proposed by Lengyel and Epstein in [3, 4]. Based on the results of Ni et al. [1, 2], we further study the steady-state problem (1.2). By the implicit function theory, we have shown that if the feed concentration is not large $\left(a^{2}<125 / 3\right)$, then the chemical concentrations of iodide $\left(\mathrm{I}^{-}\right)$and chlorite $\left(\mathrm{ClO}_{2}^{-}\right)$remain unchanged (i.e., $\left.\left(u^{*}, v^{*}\right)\right)$ when $d$ is sufficiently large (see Theorem 2.3). For the chemical concentrations of iodide $\left(\mathrm{I}^{-}\right)$and chlorite $\left(\mathrm{ClO}_{2}^{-}\right)$, we have obtained better estimates (see Theorem 3.1 and Theorem 3.2). Furthermore, by using the work of Shi [17], we have determined the change of the chemical concentrations of iodide $\left(\mathrm{I}^{-}\right)$and chlorite $\left(\mathrm{ClO}_{2}^{-}\right)$close to $\left(u^{*}, v^{*}\right)$ (see Theorem 4.1). 
Authors' contributions

All authors have made equal contributions to each part of this article. All authors read and approved the final manuscript.

\section{Author details}

${ }^{1}$ School of Mathematics, Northwest University, Xi'an, 710069, P.R. China. ${ }^{2}$ College of Mathematics and Information Science, Shaanxi Normal University, Xi'an, 710062, P.R. China.

\section{Acknowledgements}

The work is supported by the Natural Science Foundation of China (11371293). The authors would like to express their sincere thanks to the anonymous referees for their valuable suggestions which led to the improved presentation of the paper.

Received: 13 October 2015 Accepted: 13 January 2016 Published online: 26 January 2016

\section{References}

1. Ni, WM, Tang, MX: Turing patterns in the Lengyel-Epstein system for the CIMA reaction. Trans. Am. Math. Soc. 357, 3953-3969 (2005)

2. Jang, J, Ni, WM, Tang, M: Global bifurcation and structure of Turing patterns in the 1-D Lengyel-Epstein model. J. Dyn. Differ. Equ. 16, 297-320 (2004)

3. Lengyel, I, Epstein, IR: Modeling of Turing structures in the chlorite-iodide-malonic acid-starch reaction system. Science 251, 650-652 (1991)

4. Lengyel, I, Epstein, IR: A chemical approach to designing Turing patterns in reaction-diffusion system. Proc. Natl. Acad. Sci. USA 89, 3977-3979 (1992)

5. Castets, V, Dulos, E, Boissonade, J, De Kepper, P: Experimental evidence of a sustained Turing-type equilibrium chemical pattern. Phys. Rev. Lett. 64, 2953-2956 (1990)

6. De Kepper, P, Castets, V, Dulos, E, Boissonade, J: Turing-type chemical patterns in the chlorite-iodide-malonic-acid reaction. Physica D 49, 161-169 (1991)

7. Epstein, IR, Pojman, JA: An Introduction to Nonlinear Chemical Dynamics. Oxford University Press, London (1998)

8. Callahan, TK, Knobloch, E: Pattern formation in three-dimensional reaction-diffusion systems. Physica D 132, 339-362 (1999)

9. Judd, SL, Silber, M: Simple and superlattice Turing patterns in reaction-diffusion systems: bifurcation, bistability, and parameter collapse. Physica D 136, 45-65 (2000)

10. Yi, FQ, Wei, JJ, Shi, JP: Diffusion-driven instability and bifurcation in the Lengyel-Epstein system. Nonlinear Anal., Real World Appl. 9, 1038-1051 (2008)

11. Yi, FQ, Wei, JJ, Shi, JP: Global asymptotical behavior of the Lengyel-Epstein reaction-diffusion system. Appl. Math. Lett. 22, 52-55 (2009)

12. Du, LL, Wang, MX: Hopf bifurcation analysis in the 1-D Lengyel-Epstein reaction-diffusion model. J. Math. Anal. Appl. 366, 473-485 (2010)

13. Jin, JY, Shi, JP, Wei, JJ, Yi, FQ: Bifurcations of patterned solutions in the diffusive Lengyel-Epstein system of CIMA chemical reactions. Rocky Mt. J. Math. 43, 1415-1746 (2013)

14. Wei, MH, Wu, JH, Guo, GH: Turing structures and stability for the 1-D Lengyel-Epstein system. J. Math. Chem. 50 2374-2396 (2012)

15. Golubitsky, M, Schaeffer, D: Singularities and Groups in Bifurcation Theory. Springer, New York (1985)

16. Peng, $R$, Wang, MX: Spatiotemporal patterns in a reaction-diffusion model with the Degn-Harrison reaction scheme. J. Differ. Equ. 254, 2465-2498 (2013)

17. Shi, JP: Persistence and bifurcation of degenerate solutions. J. Funct. Anal. 169, 494-531 (1999)

\section{Submit your manuscript to a SpringerOpen ${ }^{\circ}$ journal and benefit from:}

- Convenient online submission

Rigorous peer review

- Immediate publication on acceptance

- Open access: articles freely available online

- High visibility within the field

- Retaining the copyright to your article 